\title{
El reto de la formación del profesorado para una enseñanza de calidad en las universidades de mayores
}

\author{
The challenge of training of teachers for quality \\ education in the universities of older \\ O desafio da formação de professores para uma \\ educação de qualidade nas universidades seniores
}

\author{
Juan Lirio Castro y Sonia Calvo Morales \\ Universidad de CAStilla La MANCha
}

\section{Resumen}

Después de más de veinte años de educación universitaria de mayores, empezamos una nueva etapa en la que nos encontramos con nuevos retos. Uno de ellos se refiere a la formación del profesorado de dichos programas, que dedicado a la educación de mayores, necesita conocer y profundizar en la especificidad del aprendizaje y la enseñanza de este grupo de edad. Si a esto le sumamos que en muchas ocasiones la formación pedagógica de dichos profesionales es escasa, este escenario se convierte en una oportunidad interesante para generar procesos de reflexión y aprendizajes, que nos permiten avanzar en la formación didáctica del profesorado de dichos programas. Pensamos además que estos procesos de formación generan traspasos, interacciones y puentes entre la docencia que estos profesores desarrollan en la Universidad convencional y la Universidad de Mayores. Este reto, por tanto, puede constituirse en una oportunidad para generar innovación educativa entre el profesorado de la universidad.
PALABRAS CLAVES: educación de mayores, formación de profesorado, innovación educativa.

\section{Abstract}

After more than twenty years of University Programmes for elderly People, We start a new phase in which we find new challenges. One of them relates to teacher training programs for those who engaged in University Programmes for elderly people. They need to know about the specific nature of learning and teaching in this age group. If we add that on many moments pedagogical training of these professionals is scarce, this scenario becomes an interesting opportunity to generate reflection and learning processes, allowing us to move forward on the didactic training of the teachers involved in these programmes. We believe that these processes generate training transfers, interactions and bridges in both teaching process (at the University and in the University Programmes for elderly people). This challenge, therefore, can serve as an opportunity to generate educational innovation among teachers of the university. 
KEYWORDS: Educative programmes for elderly people, teacher training, educational , innovation.

\section{Resumo}

Depois educação universitária seniores mais de vinte anos, iniciar uma nova etapa em que enfrentamos novos desafios. Um deles referese à formação de professores desses programas, dedicados ao ensino superior, é preciso conhecer e aprofundar a especificidade do ensino e aprendizagem dessa faixa etária. Se acrescentarmos que, em muitos casos, a formação pedagógica destes profissionais é baixo, esse cenário torna-se uma oportunidade interessante para gerar reflexão e processos de aprendizagem que nos permitem avançar a formação educacional dos professores nesses programas. Pensamos que esses processos também geram transferência de treinamento, interações e ligações entre os professores ensinando essas padrão desenvolvido na Universidade e na Universidade de Seniores. O desafio, portanto, pode se tornar uma oportunidade de gerar inovação educacional do corpo docente da universidade.

PALAVRAS-CHAVE: ensino seniores, formação de professores, a inovação educacional.

\section{Las Universidades de Mayores en España}

Aunque con una historia relativamente reciente, el origen de la educación universitaria de mayores en España se remonta ya a los años ochenta (Blázquez, 2002 y Velázquez et al., 1999). En este momento desde el tejido asociativo de mayores de Cataluña se inicia un movimiento que intenta vincular la educación permanente con la Universidad.

Posteriormente, y tras los pasos de los pioneros catalanes, comienzan a expandirse durante los años noventa los que venimos denominando Programas Universitarios de Mayores o "Universidades de Mayores" por el resto del territorio nacional. Tanto es así que en la actualidad existen más de 50 programas y alrededor de 30.000 estudiantes que siguen estos estudios. Observándose según Alonso, Lirio y Herranz (2007) varios tipos de programas universitarios de mayores:

1) Programas específicos: destinados especialmente a las personas mayores, con un plan de estudios adaptado e incluso en aulas diferentes a las utilizadas en la Universidad ordinaria.

2) Programas integrados: consistente en abrir las aulas ordinarias a los estudiantes mayores, acudiendo como un alumno más a la universidad.

3) Modelo catalán: habitualmente gestionado por los propios mayores y sus asociaciones, consisten en ciclos de conferencias sobre temas que les interesan especialmente.

4) Modelo mixto: en este tipo de programas se incluyen una parte de carácter específico y otra de de tipo integrado.

Lógicamente estos primeros años el mayor esfuerzo ha estado dedicado a la implementación de estos programas con todo lo que esto supone: diseño de los programas, búsqueda de alumnos, negociaciones con la universidad, búsqueda de financiación, creación de foros científicos, aparición de los segundos ciclos y otras modalidades formativas, creación de la asociación estatal, etc, (Lirio, Alonso, Morales y Herranz, 2006). Invirtiéndose, en consecuencia, el tiempo y los recursos en el diseño e implementación de estos estudios. Superada esta fase inicial pensamos que se debería pasar a una segunda de análisis y mejora de los programas, si pretendemos subsanar errores y sobre todo aumentar la calidad educativa de los mismos. En este sentido consideramos que un primer paso consiste en conocer bien los programas y reflexionar sobre lo que hacemos en los mismos para lo que la evaluación de los programas resulta fundamental (Lirio, Herranz y Otros, 2006). Consideramos que sin esta información cualquier intento de mejora estaría desconectado de la realidad que intenta modificar. 
En cuanto a las características generales de los programas Lirio (2008) realiza un estudio en el que a partir de la información institucional así como la guía de programas universitarios de mayores menciona las siguientes:

- La heterogeneidad de los mismos, dado que se adaptan a las instituciones y al entorno a que pertenecen.

- La mayoría son programas de tipo específico, dependientes de una universidad pública, de tres cursos de duración y una sede. - Gran parte de estos programas abordan contenidos de humanidades y culturales, siendo minoritarios los programas que abordan temas sobre la vejez o el envejecimiento.

- Casi todos los programas ofertan un programa de actividades complementarias (visitas, ciclos de conferencias, etc.).

También en este estudio se nos presentan los aspectos más positivos de estos programas así como aquellos que habría que mejorar. Entre los aspectos positivos destacan:

- Consolidación de los programas universitarios de mayores.

- Diversidad de programas para adaptarse a realidades dispares, lo que conlleva cierta originalidad y flexibilidad.

- La alta valoración que hacen los mayores tras su paso por la universidad.

- Visibilización del campo de la educación de personas mayores y contribución a la teorización e investigación en esta especialidad.

Por otra parte, entre los aspectos a mejorar o que aún no aparecen resueltos encontramos:

- Inestabilidad en la financiación (Lorenzo, 2003).

- Falta de regulación educativa.

- Falta de respaldo de la comunidad académica (Bru, 2006).

- Falta de discusión y fundamentación para poder alcanzar el reconocimiento oficial (Alfageme et al., 2003).
- Continuación de los estudios (Rojas y Ruiz, 2006).

- Ausencia de investigación (Velázquez et al.,1999, Sáez, 2005; Orte, 2006 y Lemieux, 1997).

- Son programas cuyos destinatarios "modelo" son las clases medias olvidando otros grupos como los menos favorecidos socialmente o los discapacitados entre otros (Yuni, 2000; Sánchez Martínez, 2003).

- Ausencia de trabajos sobre la perspectiva de género a pesar de que son las mujeres el público mayoritario de estos programas (Yuni, 2000).

Como puede comprobarse, los programas universitarios de mayores en un corto plazo de tiempo han conseguido instaurarse en nuestro país. Para ello han recurrido a varias estrategias que, sin duda, han tenido como denominador común la flexibilidad a la hora de adaptarse a las exigencias y realidad de las instituciones y el entorno en el que se ubicaban. En este sentido y a pesar de los retos que en la actualidad presentan, resultan interesantes como dispositivos educativos que responden a una necesidad nueva no atendida suficientemente en nuestras sociedades: la demanda de educación de las personas mayores. Sin duda aprender en esta etapa de la vida cobra un significado especial y presenta unas condiciones particulares, tanto es así que Martín (1995) apela a este aspecto, el aprendizaje en la vejez, como un área de investigación de la gerontología educativa. A continuación exploramos la particularidad del aprendizaje en esta etapa de la vida, con la intención de reflexionar sobre sus aspectos centrales.

\section{El aprendizaje en la vejez}

Antes de reflexionar sobre la especificidad o no del aprendizaje en la vejez, resulta obvio reconocer la capacidad de aprendizaje en esta etapa vital. Sin esta creencia, analizar los elementos diferenciales que influyen en el aprendizaje de las personas mayores carecería de 
sentido. Pero, lejos de controversias, no podemos dejar de reconocer que en el caso que nos ocupa, la posición de estudiante la adopta una persona mayor, que lógicamente presenta unas características harto distintas de estudiantes adolescentes, jóvenes e incluso adultos.

A nivel cognitivo, podemos recordar como nos mencionan Yuni y Urbano (2005) la diferencia entre inteligencia fluida (de carácter genético) y cristalizada (producida por el ambiente y la experiencia y que aumenta o al menos se mantiene con el tiempo). Esta distinción nos permite comprender que el aprendizaje es posible en el tiempo así como la importancia de la mediación del ambiente y la experiencia. Por tanto, la experiencia en las personas mayores se convierte en un factor que facilita y permite el aprendizaje, y en el que sin duda habremos de apoyarnos.

Otro aspecto que los autores destacan como propio de esta etapa es el pensamiento postformal. Según Martín García (1999) las características generales del pensamiento postformal son:

- El pensamiento adulto es un pensamiento divergente. El pensador postformal acepta la contradicción como un aspecto básico de la realidad. La naturaleza del pensamiento es relativa y no absoluta, incluye poder desarrollar la habilidad para sintetizar pensamientos, emociones o experiencias contradictorios dentro de un conjunto más amplio, integrado y coherente. Este pensamiento postformal no sólo permite el desarrollo de procedimientos heurísticos para resolver determinadas situaciones y problemas, sino que además permite descubrir nuevos problemas, que generalmente no tienen una única solución sino varias en las que hay que considerar tanto elementos cognitivos como emocionales, seleccionando aquellas que sean más realistas y adaptadas al contexto. También se denomina a este pensamiento como sabiduría, cuyas características básicas serían: a) la habilidad para utilizar la experiencia dentro del domino del pensamiento personal; b) el énfasis en los as- pectos pragmáticos o prácticos de la inteligencia y del conocimiento; c) énfasis sobre el contexto del problema; d) la incertidumbre como una característica de problemas y soluciones; e) la reflexión y el relativismo necesarios en cualquier tipo de juicios y acciones.

- El pensamiento adulto es un pensamiento metasistemático. Si un individuo puede observar la realidad en términos no sólo lógicos, sino relativos y contradictorios, entonces esta realidad (y el conocimiento de la misma) debe estar configurada como una estructura que adopta la forma de sistema abierto y dinámico, constituido por múltiples dimensiones que interactúan entre sí, lo que permite a los adultos contemplarla en su globalidad, de modo integral y completo. Pero además de esto, esta realidad está formada por múltiples sistemas, por tanto no es posible considerar uno único sino varios. El pensamiento postformal engloba operaciones que son aplicadas a múltiples sistemas de referencia. La cognición sobre diversos sistemas y sus interrelaciones son denominadas operaciones metasistemáticas. Y por último, el pensamiento adulto es un pensamiento contextualizado y dialéctico.

Pero además de estos aspectos referidos al pensamiento en la vejez, existen otros factores que condicionan su posición como persona en situación de aprendizaje. Uno de estos aspectos se refiere a las metas de aprendizaje. En este sentido, lejos de aprender con el objetivo de conseguir un título o vinculado al trabajo, las personas mayores quieren aprender algo que les resulte útil, es decir, que sea funcional para ellos, produciéndose así un traspaso de la utilidad que podemos denominar social a la utilidad personal. Según Lirio (2005) parece ser que la meta principal que promueve el aprendizaje en estas edades sea la necesidad de autodesarrollo, meta que se puede dividir en dos aspectos:

a) Desarrollo cognitivo, es decir, ampliar los propios conocimientos, desarrollar habi- 
lidades y manejar nuevas herramientas (nuevas tecnologías...) entre otros, con el claro objetivo de prevenir posibles dificultades o déficits posteriores (memoria, etc.).

b) Desarrollo social y personal, buscando para ello nuevos escenarios de participación, ampliando sus redes sociales, creando nuevos vínculos, dando un sentido útil a su tiempo libre, manteniéndose activos y siendo así valorados debido a sus capacidades.

En cuanto al modo en que les gusta aprender o lo que se viene denominando metodología didáctica, a pesar de la heterogeneidad del colectivo de mayores y por tanto la disparidad de preferencias con relación a la metodología didáctica, existen algunas ideas básicas que tanto la literatura como la experiencia nos muestran como fundamentales en la educación de personas mayores.

En primer lugar, resulta clave crear un clima positivo de aprendizaje. El mayor no aprenderá si no se siente aceptado, tranquilo y sin temor a equivocarse. Para ello el profesor debe apoyarles constantemente, animarles, motivarles y reconocer sus éxitos. La comunicación se convierte así en una herramienta imprescindible para el buen funcionamiento del grupo.

Una vez conseguido este buen clima, y con relación al contenido, podemos afirmar que los mayores aprenden a través de la construcción activa del conocimiento, es decir, participando. Valoran interactuar con los compañeros, debatir, y los apoyos audiovisuales (cuadros resumen, gráficos, mapas conceptuales, vídeos, etc.). A su vez en cuanto al modo de presentar los contenidos es importante para ellos exponer lo que saben del tema (activar los conocimientos previos), lo que le permite al profesor partir de sus conocimientos para llevarles poco a poco al nuevo conocimiento a aprender. Necesitan aprender nuevos conceptos, pero de un modo organizado y con un ritmo adecuado (tanto a su capaci- dad de procesamiento de la información, como a la necesidad de explicar lo que piensan del tema, aportar lo que puedan del mismo y debatir). En palabras de Sáez y Sánchez (2006) los tiempos y los ritmos para aprender son particulares. Finalmente resulta clave que se lleguen a conclusiones que sinteticen lo aprendido.

Por último, la evaluación es vivida por los alumnos con ansiedad. Las personas mayores rechazan este elemento educativo por la presión y estrés que les genera, llegando incluso a bloquear su capacidad de aprendizaje. Es muy probable que esta actitud proceda de experiencias educativas y de evaluaciones anteriores que les provocaron situaciones negativas y de fracaso, o también puede deberse a una falta de cultura de evaluación en la que nunca se revisa qué hacemos, cómo lo hacemos y por qué lo hacemos o si lo podríamos hacer de otro modo. No obstante habría que resaltar que la evaluación es una necesidad, al menos en lo que se refiere al aprendiz para que se revise qué ha aprendido, cómo lo ha aprendido y qué hay que reforzar. Así planteada, la evaluación se convertiría en un momento de reflexión necesario para dar coherencia y rigor al proceso de aprendizaje además de ofrecer una "sensación de avance" y de seriedad -ya que si después de un buen proceso educativo no existe la evaluación, los alumnos perciben un vacío o falta de cierre del proceso-- Sáez (2005) en este sentido recomienda la evaluación de tipo formativo, Lemieux (1997) plantea la autoevaluación o evaluación en parejas y Yuni y Urbano (2005) recomiendan la hetero y autoevaluación.

En nuestra opinión, lo que caracteriza realmente la educación de las personas mayores no es tanto los métodos con que les gusta aprender (que coincide con las preferencias que también se tienen en otras etapas de la vida) sino las metas que persiguen (básicamente de autodesarrollo) y la situación vital que atraviesan (el propio proceso de envejecimiento y las dificultades que esta etapa con- 
lleva) lo que les hace situarse ante el aprendizaje de un modo específico y distinto al de otras edades.

Esta realidad (el cambio de roles sociales, cambios en el procesamiento de la información, descenso de la funcionalidad, la jubilación, la crisis del nido vacío, los cambios físicos, etc.) es la que hace que las personas mayores aprendan de un modo específico y es la que nos empuja a contextualizar y desarrollar un tipo de educación diferente y necesaria para esta etapa de la vida. Y, además, esta realidad constituiría el pilar sobre que el tendrá que construirse y desarrollarse la disciplina que se encargue de la educación de personas mayores, ya se denomine gerontagogía ya gerontología educativa.

\section{La formación del profesorado de las Uni- versidades de Mayores}

Es indudable que el profesor en toda situación educativa es el factor más importanteaunque no el único- para que se produzca o no el aprendizaje. Con las personas mayores ocurre también del mismo modo (Herranz, Lirio, Alonso y Morales, 2005), y aunque no existe un modelo único de profesor adecuado para enseñar a mayores, si que podemos reflejar las preferencias que éstos manifiestan para conseguir aprender adecuadamente.

- En cuanto a los contenidos, demandan un profesor que domine la materia que imparte pero a la vez que la haga interesante. Les gusta que el profesor fundamente sus clases.

- En cuanto a lo pedagógico, que genere un buen ambiente de aprendizaje, que estructure bien las clases, que realice ejercicios prácticos, que construya conocimientos a partir de los conocimientos de los alumnos, que genere debate, que sea capaz de ponerse al nivel de los alumnos y dirigirse a ellos de un modo sencillo, sin excesivos tecnicismos.
- En cuanto a características personales, que sea un buen comunicador, que motive y sea entusiasta, que sea cercano y conocedor de la etapa vital que atraviesan los mayores, que acepte las críticas y permita la participación, que atienda a su vez las necesidades de los alumnos (conocer a los compañeros, expresarse, romper la monotonía, etc.). En definitiva valoran muy positivamente a aquellos profesores que además de una buena formación teórica y pedagógica (con una amplio y variado dominio de técnicas y recursos didácticos) sean buenos comunicadores y manejen los grupos adecuadamente, consiguiendo crear un buen ambiente de aprendizaje (aceptación, respecto, abordamiento adecuado de conflictos, etc.). A pesar de la importancia del profesor, encontramos que en la literatura específica de este campo, son pocas las referencias a los docentes que trabajan con personas mayores. Así en la guía de programas universitarios únicamente se refieren a este aspecto cuando se refiere al sistema de elección de profesores, afirmando que en cada universidad es diferente. En unas la selección de los profesores recae sobre el director del programa; en otros los que eligen son los coordinadores de área o bien son los propios profesores los que se ofrecen. No obstante, el comportamiento más generalizado es la selección por parte del director.

Por su lado Sabaté (2006) de la "Universidad Rovira i Virgili” (Tarragona) recoge en un artículo los resultados de una sencilla recogida de información que realizó al terminar el curso 2003/4 en que pidió a los alumnos de las aulas de mayores (unos 1.800) cuatro palabras que refiriesen los rasgos más significativos del que consideraban como un profesor-modelo para sus aulas. Entre las conclusiones este autor dice que los alumnos mayores esperan un profesor bien preparado para impartir la materia que le corresponde, que sea claro y transparente en la exposición, que consiga captar la atención de los alumnos y que viva lo que está explicando, además valoran la puntualidad y 
la asistencia, y manifiestan que no quieren ser tratados como niños.

Blázquez (2002) de la Universidad de Extremadura afirma que la actitud más valorada por los alumnos del Programa Universitario en sus profesores es la buena formación en la materia a impartir, seguida de la adaptación a los alumnos. La opción simpatía, es la tercera opción más relevante considerada por los alumnos a la que sigue las dotes pedagógicas del profesorado. Y si como decíamos las referencias al profesor en el contexto de los procesos educativos de las personas mayores son escasas, el tema de la formación de los profesores de las universidades de mayores es prácticamente inexistente. Dé Cassia (2010), con relación con la formación de los profesores que dan clases a los adultos mayores, indica que de los profesores entrevistados en su estudio son profesores el $72 \%$ (de enseñanza media y superior), haciendo caso omiso de la necesidad de una formación específica, aunque el $40 \%$ de ellos asistieron a un curso que se llevó a cabo sobre el trabajo con personas mayores. Finalmente podemos afirmar que únicamente Velázquez (1999) aborda esta cuestión en un artículo en el que reflexiona sobre la importancia de conocer el aprendizaje y por tanto la enseñanza de las personas mayores. En este sentido el autor afirma que algunos aspectos que deberían tener en cuenta la formación para profesores de mayores tendría que incluir tres aspectos fundamentales:

1) El aspecto humano; 2) El paradigma en el que se fundamenta la didáctica, y 3) La conceptualización del aprendizaje. También este autor llega a afirmar "En un programa Universitario de Mayores considero que es fundamental, que se tenga en cuenta tanto al profesor y al alumno, como el modelo didáctico y la concepción del aprendizaje que se defiende, todo ello como elementos que configuran el modelo de intervención didáctica que se propone...", (Velázquez, 1999; 142). Y propone la concepción del aprendizaje significativo como la más interesante para este tipo de enseñanza.
Recientemente, en el X Encuentro Nacional de Programas Universitarios de Mayores aunque se menciona la idea de la mejora de los programas universitarios de mayores únicamente se menciona al profesorado afirmando "También se podrían crear becas o ayudas, tanto para la formación como para el intercambio, que podría ser en colaboración con otras instituciones, públicas o privadas, fundamentalmente para los alumnos, pero también para los profesores de los programas", (Rodríguez, 2008: 90). Aunque no se llega a determinar qué tipo de formación ni con qué fin. Aunque Navas (2008) menciona de un modo general la necesidad de incorporar procesos de formación y reciclaje para directivos y profesorado de los Programas Universitarios -según él para conseguir adaptar estas propuestas a la nueva realidad social y a las nuevas necesidades del colectivo de personas mayores beneficiarias de los programas-, únicamente Vila (2008) se refiere directamente a la formación específica del profesorado de las universidades de mayores al recordar:

"Con el fin de mejorar la calidad de la docencia en el Programa y dado que se trata de una enseñanza diferenciada, tanto por las características del alumnado como por sus objetivos, debe ofrecerse la posibilidad de formación específica al profesorado, con el fin de dotarle de las competencias adecuadas con respecto a tal diferenciación. Así, ya este curso se programó, en el mes de octubre, un curso de formación para profesorado de adultos, impartido por especialistas pedagogos y expertos en este tipo de programas, con gran éxito de participación. Esta formación, a través del Instituto de Ciencias de la Educación, será programada cada curso, con el fin de responder a las inquietudes de este profesorado", (Vila, 2008: 198).

Aspecto al que también se suma Delgado (2005) cuando afirma que el profesor universitario debe tener, aparte de conocimientos sobre su propia materia, cierta formación en la educación de adultos. Por ello no está de más la formación permanente del profesorado en ese orden. 


\subsection{La Apuesta por la formación del profesorado de la Universidad de Mayores de Lleida}

Como hemos dicho en párrafos anteriores, la formación sigue siendo sin lugar a dudas el pilar fundamental para un proceso de enseñanza eficaz, donde el conocimiento de las claves metodológicas así como el intercambio de experiencias a través de encuentros de formación con, para y desde el profesorado, se convierte en el mejor foro de aprendizaje que existe. Sin lugar a dudas esta formación no es posible, si desde las coordinaciones o direcciones generales de los propios programas de mayores no se da una oportunidad para ponerlos en marcha. En el caso del programa de la Universidad de Lleida, existieron dos factores claves para que se diera esta situación, por un lado, la sensibilización hacia la formación de la coordinadora del programa y por otro la necesidad sentida y manifestada por el profesorado que impartía docencia en el programa de mayores. Será en diciembre de 2008 cuando un grupo de profesores del Departamento de Pedagogía, de la Universidad de Castilla-La Mancha, pone en marcha el curso ${ }^{1}$ de formación de mayores. Desde entonces hemos formado a más de 50 profesores, teniendo en cuenta sus necesidades e inquietudes pedagógicas, orientando el contenido de los programas a disponer de unos conocimientos psicopedagógicos, dirigidos a adquirir de un marco conceptual y de unos procedimientos metodológicos para:

- Transformar la información en contenidos educativos, es decir, saber qué enseñar, cómo hacerlo y evaluarlo.

- Diseñar, implementar y evaluar nuestros proyectos educativos, favoreciendo el proceso de enseñanza grupal e individual y el desarrollo personal del alumno

- Analizar, reflexionar y mejorar nuestra práctica docente.

Esta apuesta por la formación ha hecho posible crear cauces de colaboración con el profesorado de la universidad de Lleida, estableciendo foros de comunicación presencial y virtual, para la mejora del proceso formativo, de esta manera la evaluación ${ }^{2}$ se convierte en una práctica reflexiva del docente, que va mucho más allá de la mera recogida de datos, existiendo un alto nivel de satisfacción (95\%), en el desarrollo del programa, en la calidad técnica y de presentación de la materia, en el nivel de adecuación de la metodología y de la didáctica al contenido y programación de la actividades. Pero será la posibilidad de aplicar lo aprendido no sólo a los mayores sino a los alumnos jóvenes de sus respectivas titulaciones, así como la experiencia en formación en mayores, por el profesorado que imparte el curso lo que favorece el intercambio de experiencias al estar inmersos en un contexto educativo similar, los aspectos que destacan como muy positivos en los cursos formativos. En cuanto a las propuestas de mejora, establecen principalmente, la posibilidad de trabajar sobre temas monográficos para una mayor profundización del tema y una duración mayor de los cursos.

\section{La educación de mayores y la innovación educativa}

Sin duda, la educación universitaria de mayores en España es una realidad. Los mayores se han incorporado al ámbito universitario y después de una primera etapa de diseño e implantación de estos programas universitarios, deberemos pasar a otra que mejore la calidad de los mismos. En este sentido, la formación de los profesores de estos programas se constituye en un elemento crucial. Aspecto que además cobra mayor relevancia cuando un amplio número de estos profesores carece de una formación didáctica básica, aún menos específica de la educación de las personas mayores. Por ello, comentábamos que la formación de los profesores de mayores es una necesidad si queremos ajustar más y mejor la enseñanza de personas mayores a su forma de aprendizaje. Pero a la vez que una necesidad, consideramos que la aparición de los ma- 
yores en la universidad puede constituir en sí misma un motor de innovación educativa por varias razones:

\section{Ampliar la concepción que la comunidad uni- versitaria tiene sobre la misión de la Univer- sidad. Tomando conciencia de que además de las tareas tradicionales de docencia para for- mar futuros profesionales y de investigación, en la actualidad los profesores y la institución universitaria tiene que atender la vertiente de educación permanente y extensión cultural.}

Atender a la diversidad de estudiantes, al tener que adaptarse a personas con diferentes edades, recorridos vitales, experiencias laborales, niveles educativos y culturales. Tanto en las aulas específicas de las Universidades de Mayores como en aquellas que presentan un programa integrado esta cuestión es evidente ya que los mayores por definición son el grupo de edad más heterogéneo por lo que esa diversidad también se manifiesta en las formas y modos de aprender.

Avanzar hacia una enseñanza más centrada en los alumnos, atendiendo así más sus necesidades de aprendizaje, sus conocimientos previos, sus características particulares, etc. Pasando de concepciones más tradicionales y exclusivamente centradas en los contenidos y/o el profesor, a modelos educativos más sistémicos y constructivistas.

Utilizar metodologías didácticas más activas y participativas. Es decir, recurriendo a enfoques metodológicos más activos, que tengan en cuenta la multitud de variables que convergen en los escenarios educativos y que potencien tanto el aprendizaje significativo como la transferencia. Un ejemplo lo encontramos en el aprendizaje basado en problemas cuya piedra angular consiste en organizar la enseñanza, y por tanto, el aprendizaje a partir de problemas auténticos (Bejarano y Lirio, 2009).
Ampliar la formación pedagógica del profesorado, esto lógicamente si se desarrollan acciones de formación específica para los profesores de personas mayores.

Utilizar otros tipos y técnicas de evaluación, al experimentar con los mayores otras formas de evaluación (de proceso/producto; formativa....), otros tipos de evaluación (autoevaluación y coevaluación) así como otras técnicas de evaluación (elaboración de proyectos, trabajos prácticos, resolución de casos, etc.).

Innovar al pensar nuevas tareas de aprendizaje novedosas, originales y más centradas en la realidad de las personas que aprenden.

Como puede comprobarse por lo mencionado anteriormente, por distintos motivos las universidades de mayores, sin proponérselo, están contribuyendo a la innovación educativa. Por un lado la institución universitaria ha de replantearse sus fines así como la organización de sus estudios y su capacidad para dar respuesta a las nuevas demandas sociales. Por otro lado, el profesorado al tener que atender un perfil de alumnos distinto al que esta acostumbrado así como un programa de estudios con una finalidad distinta a la profesionalizadora o investigadora, se ha visto empujado a reflexionar sobre su práctica docente. Así la propia presencia de los universitarios mayores les lleva a los mismos a desplegar otros recursos y estrategias didácticas distintos a los habituales, teniendo a su vez que establecer nuevas formas de comunicación en el aula y en definitiva rediseñar su programación didáctica, lo que implica adoptar una posición diferente como profesor. Sin duda, todos estos aspectos están contribuyendo a que el profesorado se aproxime a concepciones del aprendizaje y la enseñanza más constructivistas. Y también a interesarse más sobre su rol como docente, reflexionando sobre su práctica en estas aulas (Morales, 2009) así como intentando adaptar su conocimiento sobre la materia a la 
forma de aprender de estos alumnos. En este sentido creemos que una interesante línea de investigación vendría de la profundización en la forma de enseñanza de las distintas materias que se imparten en los programas universitarios de mayores 3 así como de la elaboración de materiales didácticos adaptados a esta etapa evolutiva y forma característica de aprender. Un ejemplo lo encontramos en el trabajo realizado por Morales (2007 y 2009) en el que nos presenta un material didáctico sobre informática adaptado a las características de los alumnos mayores y su forma de aprender. Bru (2006, 251) afirma que "Los métodos y ejemplos de innovación educativa y las buenas prácticas, en la formación permanente en general y en estudios universitarios para mayores en concreto son ejes centrales de esta dimensión internacional de los PUMP".

Quizás esta afirmación nos debiera llevar a pensar que la mejora de los programas universitarios de mayores pasa por la innovación educativa, la formación del profesorado y la profundización en el aprendizaje y la enseñanza específica de este grupo de edad. Sin duda afirmamos que el reto inmediato de estos programas consiste en aumentar el rigor en su desarrollo, la evaluación de sus propuestas, la investigación y teorización específica de este ámbito disciplinar, así como impulsar la innovación y la formación de su profesorado.

Sólo desde una apuesta decidida que mejore estos aspectos, conseguiremos mejorar aún más nuestros programas universitarios de mayores y consolidar esta propuesta como una realidad de educación permanente en la Universidad.

\section{Conclusiones}

La principal conclusión a la que llegamos tras el análisis de los programas, es la ausencia de formación específica del profesorado de las universidades de mayores. También constatamos como la propia idiosincrasia de estos programas, ha generado cambios metodoló- gicos que apuntan hacia la innovación educativa al tener que responder el profesorado de estos estudios a un perfil de alumno con necesidades, demandas y formas de aprender muy diferentes al acostumbrado en entornos académicos. Esta situación ha llevado al profesorado a tener que diseñar la enseñanza con personas mayores teniendo en cuenta su modo característico de aprender y desplegando a su vez una variedad de recursos y metodologías didácticas que se ajustaran a estos objetivos.

Recordemos que estamos ante el grupo de edad más heterogéneo del ciclo vital, lo que implica pluralidad en las concepciones de aprendizaje y diversidad en los modos de aprender y de entender la realidad. Sin duda, estos aspectos inherentes a la educación en esta etapa de la vida lleva y va a seguir llevando a los profesores que en ella laboran a la búsqueda de modelos de enseñanza-aprendizaje más integradores y constructivistas, al tener que atender a una multiplicidad de perspectivas, intereses, concepciones y modos de aprender de los alumnos mayores.

Sin duda, la aparición de la educación universitaria en España está generando en sí misma la flexibilidad en los planteamientos educativos en la institución universitaria, además de en la forma de diseñar y desarrollar la docencia, aspecto que si sabe aprovecharse adecuadamente nos permitiría iniciar un diálogo y los cimientos de una formación pedagógica más sólida que permitiera al profesorado universitario adaptarse a este tipo de alumnos y, por ende, desarrollar enfoques metodológicos que atiendan la diversidad que en general se da en los contextos educativos. De ser así, lógicamente el profesorado universitario aumentará su formación teórica y metodológica didáctica, lo que le permitirá un mayor despliegue en sus formas de actuar como docente reflexivo y estratega, trasladando inevitablemente su modo de ser como profesor a todos los escenarios educativos en que desarrolle su trabajo lo que también incluiría la Universidad ordinaria. 


\section{Referencias bibliográficas}

Alfageme, A, Cabedo, S. y Escuder, P. (2003): Los programas universitarios para mayores como política social. En VII Encuentro Nacional de Programas Universitarios para Mayores, Políticas sociales, educativas y financiación de la formación universitaria de personas mayores y su proyección social. Madrid: Ministerio de Trabajo y Asuntos Sociales-IMSERSO.

Alonso, D, Lirio, J. y Herranz, I. (2007): Los beneficios de la participación en la Universidad de Mayores. En D. Alonso, J. Lirio, J. y P. Mairal. (Coords), Mayores activos: Teoría, Experiencias y reflexiones en torno a la participación social, pp. 157-183. Madrid, Arjé.

Bejarano, M.․․ T. y Lirio, J. (2009): La utilización de problemas auténticos en la enseñanza superior. En A. Escribano y A. Del Valle (Coord.), El Aprendizaje Basado en Problemas: Una propuesta metodológica en la educación superior. Madrid: Narcea.

Blázquez, F. (2002): Los mayores, nuevos alumnos de la Universidad. Revista Interuniversitaria de Formación del Profesorado, 45, 89-105.

Bru, C. (2006): La dimensión internacional de los programas universitarios de mayores. En C. Orte (Coord.), El aprendizaje a lo largo de toda la vida. Los programas universitarios de mayores, pp. 215-261. Madrid: Dykinson.

De Cássia, R. (2010): Da educação que o sistema faz à educação que o idoso necessita e quer: um espaço discente e docente de qualidade para a terceira idade. Palabras Mayores, 4. (En línea):<http://pergamo.pucp. edu.pe/palabrasmayores>. (Consulta del 16 de Marzo de 2010).

Delgado, A. A. (2005): Ventajas e inconvenientes de la diversidad en el alumnado de los programas de mayores: Análisis de una experiencia. Actas del I Congreso Iberoamericano de Experiencias Educativas en Adultos Mayores. Lima, Universidad Católica Pontificia de Lima.

Herranz, I; Lirio, J; Alonso, D. y Morales, S. (2005): El papel decisivo del profesor en la valoración por parte de los alumnos del contenido de las materias del programa universitario de mayores. Actas del I Congreso Iberoamericano de Experiencias Educativas en Adultos Mayores. Lima, Universidad Católica Pontificia de Lima.

Lemieux, A. (1997): Los programas universitarios para mayores. Enseñanza e investigación. Madrid: IMSERSO.

Lirio, J. (2008): La Gerontología Educativa en España: Realidad sociodemográfica y concepciones de apren- dizaje de los alumnos de la Universidad de Mayores "José Saramago" de la sede de Talavera de la Reina de la Universidad de Castilla La Mancha. (En línea): <http://eprints.ucm.es/8315/1/ T30671.pdf>. (Consulta del 20 de Febrero de 2010).

Lirio, J. (2005): Claves para comprender el aprendizaje de las personas mayores. Revista Electrónica de Psicogerontología Tiempo 17, 1-7, noviembre. (En línea): <www.psiconet.com/tiempo>. (Consulta del 10 de febrero de 2006).

Lirio, J; Alonso, D; Morales, S. y Herranz, I. (2006): La satisfacción como motor de participación y ciudadanía en la educación universitaria de personas mayores. En C. Zorita; N. Yuste y J. J. Gázquez, Mayores en la Universidad: derecho, necesidad, satisfacción, pp 155-166. Almería, Servicio de publicaciones de la Universidad de Almería.

Lirio, J; Herranz, I; y Otros. (2006): La evaluación como instrumento de calidad y mejora en las universidades de mayores: la evaluación del programa específico de la universidad de mayores José Saramago. En Mํa․ A. Holgado y M. ${ }^{\underline{a}}$ T. RamosS, (Dir); VIII Encuentro Nacional de Programas Universitarios para Mayores. Una apuesta por el aprendizaje a lo largo de la vida, pp. 61-70. Madrid, Ministerio de Trabajo y Asuntos Sociales.

Lorenzo, J. A. (2003): El marco educativo de la formación universitaria de las personas mayores: La necesidad de una política educativa. En VII Encuentro Nacional de Programas Universitarios para Mayores: Políticas sociales, educativas y financiación de la formación universitaria de personas mayores y su proyección social. Madrid: Ministerio de Trabajo y Asuntos Sociales.

Martín, A. V. (1999): Más allá de Piaget: Cognición adulta y educación. Teoría de la Educación, 11, 127157.

Martín, A. V. (1995): Objeto y ámbitos de investigación en Gerontología Educativa. Pedagogía Social, 12, 7-21.

Morales, S. (2009): La asignatura de Informática en la Universidad de Mayores: La Universidad de Mayores "José Saramago" de la Universidad de Castilla la Mancha. Tesis doctoral. Universidad Complutense.

Morales, S. (2007): Fórmate tecleando. Toledo: Universidad de Castilla La Mancha.

Morales, S; Alonso, D; Lirio, J. y Herranz, I. (2006): Variables que influyen en el proceso de enseñanza-aprendizaje en la asignatura de Informática de la Universidad de Mayores José Saramago de Talavera de la Reina. 
En C. Zorita, C; N. Yuste y J. J. Gázquez. Mayores en la Universidad: derecho, necesidad satisfacción. Almería, Servicio de publicaciones de la universidad de Almería.

Navas, J. C. (2008): Hacia un nuevo diseño de los programas educativos para mayores. En $\mathrm{M}^{\mathrm{a}} \mathrm{C}$. Palmero, (coord.), Formación universitaria de personas mayores y promoción de la autonomía personal. Políticas socioeducativas, metodologías e innovaciones. Burgos: Universidad de Burgos.

Orte, C. (Coord.). (2006): El aprendizaje a lo largo de toda la vida. Los programas universitarios de mayores. Madrid: Dykinson.

Rojas, C. y Ruiz, D. (2006): 'Acción Social' de mayores universitarios. En $\mathrm{M}^{\underline{a}}$ Holgado y $\mathrm{M}^{\underline{a}} \mathrm{~T}$. Ramos (Dir.), VIII Encuentro Nacional de Programas Universitarios para Mayores: Una apuesta por el aprendizaje a lo largo de la vida. Madrid: Ministerio de Trabajo y Asuntos Sociales.

Rodríguez, A. (2008): Retos de la Asociación Estatal de Programas Universitarios para Personas Mayores. En $\mathrm{M}^{\mathrm{a}}$ C. Palmero, (coord.), Formación universitaria de personas mayores y promoción de la autonomía personal. Políticas socioeducativas, metodologías e innovaciones. Burgos: Universidad de Burgos.

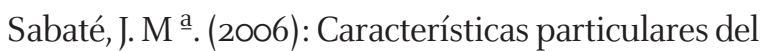
profesor-modelo de las A.E.U.G.G. de la Universidad Rovira i Virgili: Una sencilla experiencia para una primera aproximación. En $\mathrm{M}^{\underline{a}}$. Holgado y $\mathrm{M}^{\underline{a}}$ T. Ramos (Dir.), VIII Encuentro Nacional de Programas Universitarios para Mayores: Una apuesta por el aprendizaje a lo largo de la vida. Madrid: Ministerio de Trabajo y Asuntos Sociales.

Sáez, J. (2005): Gerontagogía: Intervención socioeducativa con personas mayores. En S. Pinazo y M. Sánchez (Dir.): Gerontología. Actualización, innovación y propuestas. Madrid: Pearson Educación.

Sáez, J. y Sánchez, M. (2006): La educación de personas mayores en la sociedad de la información y el conocimiento. En C. Orte (Coord.), El aprendizaje a lo largo de toda la vida. Los programas universitarios de mayores. Madrid: Dykinson.

Sánchez Martínez, M. (2003): La semántica en la terminología en la educación de personas mayores. En J. SÁEZ. Educación y aprendizaje en las personas mayores. Madrid: Dykinson.

Velázquez, M. (1999): La formación de formadores para los Programas Universitarios de Mayores. Escuela Abierta, 3, 133-162.

Velázquez, M, Fernández, C; Holgado, Ṃㅗ A, Guirao, M. y Sánchez, M. (1999): Guía de Programas Universitarios de Personas Mayores. Madrid: Ministe- rio de Trabajo y Asuntos Sociales-IMSERSO.

Vila, N. (2008): Los criterios de calidad en los Programas Universitarios para Mayores. En $\mathrm{M}^{\mathfrak{a}} \mathrm{C}$. Palmero, (coord.), Formación universitaria de personas mayores y promoción de la autonomía personal. Políticas socioeducativas, metodologías e innovaciones. Burgos: Universidad de Burgos.

Yuni, J. A. (2000): El mito del eterno retorno. Educación, subjetividad y adultos mayores. En S. Duschatzky, Tutelados y asistidos. Programas sociales, políticas públicas y subjetividad. Buenos Aires: Paidós.

Yuni, J. A. y Urbano, C. A. (2005): Educación de adultos mayores. Teoría, investigación e intervenciones. Córdoba: Brujas.

\section{Notas}

${ }^{1}$ El contenido de los cursos de formación dirigidos al profesorado del programa de la universidad de mayores de la universidad de Lleida puede consultarse en la siguiente dirección: http://www.ice.udl.cat/upu/ programes/programes2009-2010/Uo472.pdf.

2 Resultados extraídos de las memorias de evaluación internas ( evaluación , 2007,2008,2009)del Instituto de Ciencias de la Educación de la Universidad de Lleida..

3 Aspecto que viene siendo abordado y recogido en los distintos Encuentros Nacionales de Programas Universitarios para Mayores, como por ejemplo la contribución sobre el aprendizaje en la materia de Informática que presentan Morales, Alonso, Lirio y Herranz, 2006.

DIRECCIÓN DE LOS AUTORES: Juan Lirio Castro y Sonia Morales Calvo. Universidad de Castilla la Mancha. Departamento de Pedagogia. Avd/ Real Fábrica de la Seda s/n 45600.Talavera de la Reina. Correos electrónicos: sonia.morales@uclm.es; juan.lirio@uclm.es

Fecha de recepción del artículo: 29.III.2010

Fecha de revisión del artículo: 06.IV.2010

Fecha de aceptación del artículo: 20.IX.2010

\section{COMO CITAR ESTE ARTÍCULO:}

Lirio Castro, J. y Morales Calvo, S. (2011) “El reto de la formación del profesorado para una enseñanza e calidad en las universidades de mayores", en Pedagogía Social. Revista Interuniversitaria, 19, pp. 155-166. 